\title{
Catquest-9SF functioning over a decade - a study from the Swedish National Cataract Register
}

\author{
Mats Lundström ${ }^{1 *}$ (D), Maria Kugelberg ${ }^{2,3}$, Per Montan ${ }^{2,3}$, Ingela Nilsson ${ }^{4}$, Madeleine Zetterberg $^{5}$,
} Konrad Pesudovs ${ }^{6}$ and Anders Behndig ${ }^{7}$

\begin{abstract}
Background: The Swedish National Cataract Register (NCR) collects data on cataract surgery outcomes during March, including patient-reported outcomes using the Catquest-9SF questionnaire for over 11 years. Previous studies from NCR have shown that the preoperative visual acuity has improved over time. The main purpose of this study was to evaluate the Catquest-9SF Rasch scoring performance in this changing environment. A second purpose was to describe clinical data over the same period for those who completed the questionnaire.

Methods: The performance of the Catquest-9SF was analysed by a separate Rasch analysis for each year, resulting in a preoperative and postoperative score for each participating patient in the annual cohorts. The clinical data and questionnaire scoring were analysed for each year in the period 2008-2018 inclusive.
\end{abstract}

Results: Data were available for 42,023 eyes for 11 annual cohorts (2008-2018). The psychometric properties of the questionnaire were stable during the study period. Person separation (precision) for the whole period was 2.58 and varied between 2.45 and 2.72. The person reliability was 0.87 and varied between 0.86 and 0.88 . The targeting of question difficulty to person ability became less accurate over time meaning that the item activities became easier to carry out without difficulty. The average targeting for the whole period was -2.06 and changed from -1.92 in 2008 to -2.31 in 2018. The person score improved both before surgery and after surgery, indicating that patients are undergoing surgery at a more able level and getting better outcomes. The average improvement by surgery decreased from 3.41 logits in 2008 to 3.21 logits in $2018(p=0.003)$.

Over time, patient age decreased from 75 to 74 years $(p<0.001)$ and the proportion of women decreased from 63.9 to $57.9 \%(p<0.001)$. The mean preoperative visual acuity in both the operated eye and the better eye improved over time ( 0.47 to $0.40 \log M A R, p<0.001$ and 0.22 to $0.19 \log M A R, p<0.001$, respectively), as did the mean postoperative visual acuity in the operated eye (0.14 to $0.09 \operatorname{logMAR}, p<0.001)$.

Conclusions: The Catquest-9SF retained stable psychometric properties over this 11-year period although more recent cohorts included slightly younger patients with somewhat better vision.

Keywords: Cataract extraction, Outcomes, Questionnaire, Patient-reported outcomes, Rasch analysis

\footnotetext{
* Correspondence: mats.lundstrom@karlskrona.mail.telia.com

'Department of Clinical Sciences/Ophthalmology, Faculty of Medicine, Lund University, Trossögatan 4, 37137 Karlskrona, Lund, Sweden

Full list of author information is available at the end of the article
}

(C) The Author(s). 2020 Open Access This article is licensed under a Creative Commons Attribution 4.0 International License, which permits use, sharing, adaptation, distribution and reproduction in any medium or format, as long as you give appropriate credit to the original author(s) and the source, provide a link to the Creative Commons licence, and indicate if changes were made. The images or other third party material in this article are included in the article's Creative Commons. licence, unless indicated otherwise in a credit line to the material. If material is not included in the article's Creative Commons licence and your intended use is not permitted by statutory regulation or exceeds the permitted use, you will need to obtain permission directly from the copyright holder. To view a copy of this licence, visit http://creativecommons.org/licenses/by/4.0/ The Creative Commons Public Domain Dedication waiver (http://creativecommons.org/publicdomain/zero/1.0/) applies to the data made available in this article, unless otherwise stated in a credit line to the data. 


\section{Background}

The Swedish National Cataract Register (NCR) [1] has used the Catquest-9SF questionnaire [2] regularly since 2008. The NCR collects data on outcomes of cataract surgery during March each year. Catquest-9SF is a Rasch scaled 9-item questionnaire measuring activity limitations in daily life due to poor vision because of cataract and is used by the NCR because of its documented responsiveness in cataract surgery $[3,4]$. It has been translated to many languages and validated through Rasch analysis [5] and is recommended by the International Consortium of Health Outcomes Measurement [6] and the European Registry of Quality Outcomes for Cataract and Refractive Surgery [7].

As of 2018, the Catquest-9SF has remained unchanged since 2008.

It comprises 9 items (A, B, C1-C7) with 4 response options and the possibility to check "cannot decide" which is treated as missing data. Items $\mathrm{A}$ and $\mathrm{C} 1-\mathrm{C} 7$ are concerned with difficulty, and item B with satisfaction. Response options are coded 4 for "very great difficulty/ very dissatisfied", 3 for "great difficulty/fairly dissatisfied", 2 for "some difficulty/fairly satisfied" and 1 for "no difficulty/very satisfied". The clinical impression during these 11 years is that the indication for cataract surgery has slowly drifted towards better preoperative visual acuity. This change in preoperative visual acuity has been documented for three national registries where the Catquest9SF is used: Sweden, the Netherlands and Malaysia [8]. Furthermore, the availability of cataract surgery in Sweden has improved over this period, with an increase in annual volume from about 75,000 procedures in 2008 to 133,000 in 2017 [9]. The question remains as to whether the psychometric characteristics of Catquest-9SF have been optimal for the changing clinical patient cohorts during this period.

The purpose of this study was to evaluate the Catquest-9SF Rasch scoring performance for cohorts with gradually increasing visual acuity and changing risk factors (ocular comorbidity, difficult surgery because of small pupil, white cataract, and previous eye surgery) [8] over 11 years. We think this is of general interest, given the widespread use of the questionnaire, and also of specific interest to cataract surgeons who may want to adopt the questionnaire.

\section{Methods}

Surgeons and clinics are invited by the NCR to participate in March every year on a voluntary basis. About $80 \%$ of all clinics reporting preoperative data to NCR also take part in the outcome data collection by reporting data on consecutive surgeries for March. Preoperative data include age, sex, visual acuity of both eyes, previous cataract surgery in the fellow eye, and ocular co-morbidities (age-related macular degeneration, glaucoma, diabetic retinopathy, and "other" sightthreatening eye disease) in the operated eye. Visual acuity values in original decimal notation were transformed to $\log$ MAR values for the statistical analysis. Surgical data include laterality (eye), perioperative difficulties (small pupil with need of stretching, white or dense cataract with need of staining, need of capsular hooks and capsular tension ring), type of surgery, type of intra-ocular lens (IOL) and surgical complication (posterior capsule rupture). Outcome data include preoperative $\mathrm{K}$ values, axial length, IOL calculation formula, visual acuity, planned refraction and refractive outcome (refraction).

Most of the clinics reporting outcome data during March also use the Catquest-9SF questionnaire (Fig. 1) [2]. Patients are asked to fill in the Catquest-9SF on a voluntary basis before cataract surgery and 3 months after surgery. The preoperative questionnaire is completed on paper at the clinic after surgery has been decided on, either during the preoperative examination or on the day of surgery. Patients who are unable to understand the questionnaire or do not understand Swedish are excluded from this part of the outcome study. The postoperative questionnaire is completed at home as a postal paper form with a prepaid envelope or on the web using unique per-patient login credentials. More than $90 \%$ of patients operated during March in participating clinics completed the preoperative questionnaire, and between 70 and $80 \%$ of these patients returned the postoperative questionnaire.

\section{Rasch analysis}

Precision is a crucial characteristic of all measurement tools. In Rasch analysis, precision is calculated as person separation and person reliability. These entities should have values above 2.0 and 0.80 , respectively, to show good precision. This means an ability to separate the respondents in at least three strata of visual function. These precision values provide an evaluation of how the questionnaire performs along the continuum of raw score measure for the whole test. The person separation reflects the number of levels of ability that the questionnaire can separate the respondents into, while the person reliability is a measure of the questionnaire's internal consistency. Targeting is a measure of how well the difficulty of the items matches the ability of the patients to endorse the items.

In a Rasch analysis, each item is given a Rasch score for the response options. A mean Rasch score for each item can then be calculated based on the scoring of the response options, corresponding to the difficulty of the item for the study cohort. A negative Rasch item score signifies a more difficult item to endorse, while a positive 
A. Do you find that your sight at present in some way causes you difficulty in your everyday life?

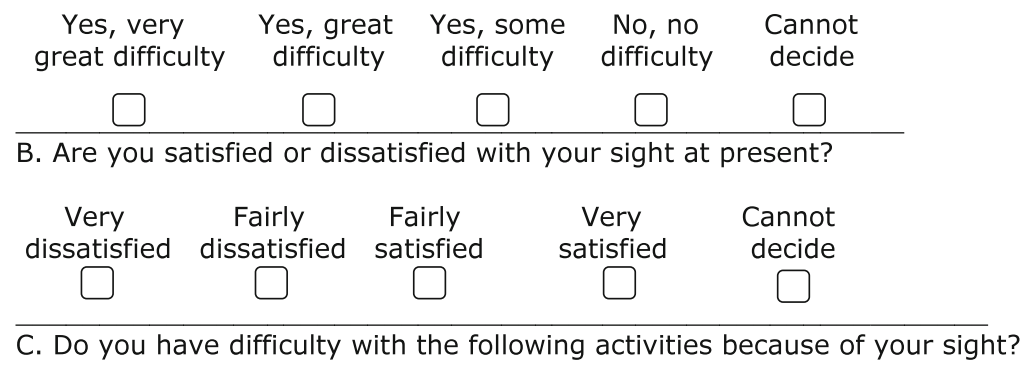

If so, to what extent? In each row place just one tick in the box which you think best corresponds to your situation.

\begin{tabular}{|c|c|c|c|c|c|}
\hline & $\begin{array}{l}\text { 'es, very } \\
\text { great } \\
\text { difficulty }\end{array}$ & $\begin{array}{l}\text { Yes, great } \\
\text { difficulty }\end{array}$ & $\begin{array}{l}\text { Yes, some } \\
\text { difficulty }\end{array}$ & $\begin{array}{l}\text { No, no } \\
\text { difficulty }\end{array}$ & $\begin{array}{l}\text { Cannot } \\
\text { decide }\end{array}$ \\
\hline $\begin{array}{l}\text { Reading text in } \\
\text { newspapers }\end{array}$ & & & & & \\
\hline $\begin{array}{l}\text { Recognising the } \\
\text { faces of people } \\
\text { you meet }\end{array}$ & & & & & $\square$ \\
\hline $\begin{array}{l}\text { Seeing the prices } \\
\text { of goods when } \\
\text { shopping }\end{array}$ & & 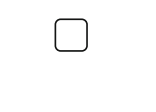 & $\square$ & $\square$ & $\square$ \\
\hline $\begin{array}{l}\text { Seeing to walk } \\
\text { on uneven surfaces, } \\
\text { e.g. cobblestones }\end{array}$ & s, & & & & \\
\hline $\begin{array}{l}\text { Seeing to do } \\
\text { handicrafts, } \\
\text { woodwork etc. }\end{array}$ & & & $\square$ & $C$ & \\
\hline $\begin{array}{l}\text { Reading subtitles on } \\
\text { TV }\end{array}$ & on $\square$ & & $\square$ & $\square$ & \\
\hline $\begin{array}{l}\text { Seeing to engage } \\
\text { in an activity/hobby } \\
\text { that you are } \\
\text { interested in }\end{array}$ & y $\square$ & 0 & 0 & 0 & $\square$ \\
\hline
\end{tabular}

Thank you very much for taking part.

Fig. 1 The English version of the Catquest-9SF form

value indicates the opposite. This is related to the raw score polarity (with 4 meaning very great difficulty and 1 meaning no difficulty). In Rasch analysis, item scoring and person scoring are calculated and analysed on the same single linear scale that uses a logit (natural logarithm of the odds ratio) unit.

\section{Statistical analysis}

Rasch analysis was performed using version 4.2.0 of Winsteps (Chicago, IL, USA). A Rasch analysis was performed independently for each annual data cohort. Statistical analyses of clinical data and Rasch person scores were performed using version 25.0 of IBM SPSS Statistics (IBM Corp., Armonk, NY, USA). Testing included bivariate Pearson correlation, t-tests, and linear regression. Trends over time were analysed with a Chi-squared test for categorical data and ANOVA for continuous data. A $p$-value of $<0.05$ was considered significant.

The study adhered to the tenets of the Declaration of Helsinki. The Swedish Data Inspection Board approved the data collection in the NCR and an ethics vetting board approved the collection of information in the Catquest-9SF questionnaire.

\section{Results}

The number of follow-up cases versus number of completed preoperative and postoperative Catquest-9SF is shown in Table 1. 


\section{Patients}

Patient characteristics in terms of age, sex, preoperative visual acuity, first- or second-eye surgery and postoperative visual acuity in the annual cohorts are shown in Table 2. The mean follow-up time in each year varied between 31 and 41 days with an overall mean of 36 days.

Preoperative visual acuity and age in groups are outlined in Table 3.

\section{Description of data}

The annual precision values together with the number of completed questionnaires and targeting are shown in Table 4 .

For the whole study period, the person separation was 2.58 and the person reliability was 0.87 . There was a tendency of misfit for item C4 (Seeing to walk on uneven ground) and item C2 (Recognizing the faces of people you meet). For item $\mathrm{C} 4$ and item $\mathrm{C} 2$, fit statistics showed Infit mean square-Outfit mean square of 1.27-1. 42 and 1.38-1.33, respectively. For the other 7 items, Infit mean square varied between 0.70 and 1.08 while Outfit mean square varied between 0.72 and 1.03 . The average targeting for the whole study period was -2.06 , the preoperative targeting was -0.45 logits and the postoperative targeting was -3.61 logits. Differential item functioning (DIF) was tested for sex and surgery year. There was no DIF over 0.5 logits for any item versus these two parameters. A mean score for each item is shown for all study years in Fig. 2.

The pattern of the average item Rasch scoring over time is shown in Fig. 3, revealing how the level of difficulty for each item varied during the study period.

Table 1 Number of cases with follow-up data and completed Catquest-9SF during March 2008 to 2018 inclusive

\begin{tabular}{lll}
\hline Surgery year & $\begin{array}{l}\text { Number of patients } \\
\text { with follow-up data }\end{array}$ & $\begin{array}{l}\text { Number (\%) of } \\
\text { patients with } \\
\text { completed } \\
\text { Catquest-9SF } \\
\text { before and after } \\
\text { surgery }\end{array}$ \\
\hline 2008 & $845(65.2)$ \\
2009 & 1296 & $3023(43.4)$ \\
2010 & 6960 & $3815(58.3)$ \\
2011 & 6549 & $3514(54.4)$ \\
2012 & 6463 & $3716(60.7)$ \\
2013 & 6118 & $3666(56.3)$ \\
2014 & 6512 & $4601(57.8)$ \\
2015 & 7954 & $4484(66.0)$ \\
2016 & 6796 & $4472(63.6)$ \\
2017 & 7031 & $5312(61.5)$ \\
2018 & 8636 & $4575(66.2)$ \\
Total & 6908 & $42,023(59.0)$ \\
\hline
\end{tabular}

Figures 2 and 3 show that items A, B and C1 change over time towards more negative Rasch item scoring which means that they are easier to endorse for the patients. The mean preoperative and postoperative Rasch person score over time is shown in Fig. 4. The Rasch person score varied between -6.45 and 5.92 in this study. A more negative value signifies less difficulty in carrying out the requested item activities in daily life. The average improvement (difference between preoperative and postoperative Rasch person score) by surgery decreased from 3.41 logits in 2008 to 3.21 logits in 2018 $(p=0.003)$.

\section{Analyses}

The bivariate Pearson correlation coefficient between preoperative Rasch person score and visual acuity (logMAR) in the operated eye, was $-0.213(p<0.01)$ for the whole period, varying between -0.165 and $-0.222(p<$ 0.01 ) for each year. The bivariate Pearson correlation coefficient between preoperative Rasch person score and visual acuity $(\log M A R)$ better eye, was $-0.283(p<0.01)$ for the whole period. For each year, it varied between -0.184 and $-0.309(p<0.01)$.

Single variable analyses of preoperative Rasch person score showed a significantly higher mean score (i.e., more perceived difficulty) for women compared to men ( -0.22 vs. $-0.68, p<0.001$, t-test), for patients with an ocular comorbidity compared to those with no ocular comorbidity $(-0.19$ vs. $-0.53, p<0.001$, t-test), for firsteye surgery compared to second-eye surgery $(-0.20$ vs. $-0.72, p<0.001$, t-test) and for patients with surgical difficulty compared to those with no surgical difficulty ( -0.06 vs. $-0.43, p<0.001$, t-test). The differences were statistically significant for every year in all analyses, except for 2008 in the sex comparison and for 3 out of the 11 years in the surgical difficulty analysis. The bivariate Pearson correlation coefficient between preoperative Rasch person score and age was $-0.046(p<0.01)$ for the whole period. When age was dichotomized into $<75$ and $\geq 75$, the younger age group had a significantly higher preoperative Rasch person score $(-0.30$ vs. -0.50 , $p<0.001$, t-test). This difference existed for analysis of each single study year but was not always statistically significant (data not shown). Table 5 shows a linear stepwise regression analysis with preoperative person Rasch score as dependent variable and all other parameters included as independent variables. All independent variables were significantly related to the preoperative person Rasch score.

In Table 5, unstandardized beta shows the size of change of the dependent variable for every 1-unit change of the independent variable. To interpret this correctly, it is important to remember that improvement of the dependent variable means a more 
Table 2 Number of patients, preoperative characteristics, and postoperative visual acuity

\begin{tabular}{lllllllll}
\hline Year & $\begin{array}{l}\text { Number of } \\
\text { patients }\end{array}$ & $\begin{array}{l}\text { Female } \\
\text { (\%) }\end{array}$ & $\begin{array}{l}\text { Mean age } \\
\text { (years) }\end{array}$ & $\begin{array}{l}\text { 2nd eye surgery } \\
\text { (\%) }\end{array}$ & $\begin{array}{l}\text { Mean preoperative } \\
\text { BCVA operated eye } \\
\text { (logMAR) }\end{array}$ & $\begin{array}{l}\text { Mean preoperative } \\
\text { BCVA better eye } \\
\text { (logMAR) }\end{array}$ & $\begin{array}{l}\text { Ocular co- } \\
\text { morbidity } \\
\text { (\%) }\end{array}$ & $\begin{array}{l}\text { Mean post-operative } \\
\text { BCVA, operated eye }\end{array}$ \\
(logMAR)
\end{tabular}

$B C V A=$ best corrected visual acuity; logMAR = logarithm of the minimum angle of resolution

a Ocular comorbidity includes age-related macular degeneration, glaucoma, diabetic retinopathy, and "other" sight- threatening ocular comorbidity

${ }^{\mathrm{b}}$ Chi-squared test

'One-way ANOVA

negative value and that a negative beta means a decrease and a positive beta indicates an increase of the dependent variable.

The same analysis was also conducted for each single year cohort separately. Preoperative visual acuity in the better eye and age were both significantly related to the preoperative person Rasch score in all single study years. Ocular co-morbidity, second-eye surgery, and sex were also significantly related to the preoperative person Rasch score in all years except for ocular comorbidity in 2009 and second-eye surgery and sex in 2008. Surgical difficulty was only significantly related to the preoperative person Rasch score in 2016.

The bivariate Pearson correlation coefficient between postoperative Rasch person score and postoperative visual acuity $(\log M A R)$ in the operated eye, was -0.355 $(p<0.001)$ for the whole period and varied between -0.292 and $-0.417(p<0.001)$ for each year. Table 6 shows a linear stepwise regression analysis with postoperative person Rasch score as dependent variable and preoperative characteristics and capsule complication included as independent variables. Capsule complication

Table 3 Visual acuity in two groups and age in two groups per year

\begin{tabular}{|c|c|c|c|c|c|c|}
\hline Year & $\begin{array}{l}\text { Preoperative } \\
\text { visual acuity, } \\
\text { logMAR } \geq 0.0 \\
(\geq 20 / 20) \\
N(\%)\end{array}$ & $\begin{array}{l}\text { Preoperative } \\
\text { visual acuity, } \\
\text { logMAR } \geq 0.3 \\
(\geq 20 / 40) \\
N(\%)\end{array}$ & $\begin{array}{l}\text { Age } \leq 65 \text { years } \\
\mathrm{N}(\%)\end{array}$ & $\begin{array}{l}\text { Age } \geq 66 \text { years } \\
N(\%)\end{array}$ & $\begin{array}{l}\text { Missing data } \\
\mathrm{N}(\%)\end{array}$ & $\begin{array}{l}\text { Total number } \\
\text { of patients }\end{array}$ \\
\hline 2008 & $10(1.2)$ & $368(43.6)$ & $131(15.5)$ & 714 (84.5) & 0 & 845 \\
\hline 2009 & $24(0.8)$ & $1252(41.4)$ & $427(14.1)$ & 2582 (85.4) & $14(0.5)$ & 3023 \\
\hline 2010 & $28(0.7)$ & 1517 (39.8) & 607 (15.9) & 3208 (84.1) & 0 & 3815 \\
\hline 2011 & $24(0.7)$ & $1658(47.2)$ & $532(15.1)$ & 2969 (84.5) & $13(0.4)$ & 3514 \\
\hline 2012 & $52(1.4)$ & $1775(47.8)$ & $529(14.2)$ & 3145 (84.6) & $42(1.1)$ & 3716 \\
\hline 2013 & $55(1.5)$ & $1853(50.5)$ & $558(15.2)$ & 3073 (83.8) & $35(1.0)$ & 3666 \\
\hline 2014 & $62(1.3)$ & 2261 (49.1) & 641 (13.9) & 3959 (86.0) & $1(0)$ & 4601 \\
\hline 2015 & $50(1.1)$ & $2241(50.0)$ & $570(12.7)$ & 3879 (86.5) & $35(0.8)$ & 4484 \\
\hline 2016 & $72(1.6)$ & $2438(54.5)$ & $558(12.5)$ & 3875 (86.7) & $39(0.9)$ & 4472 \\
\hline 2017 & $77(1.4)$ & 2995 (56.4) & $561(10.6)$ & 4750 (89.4) & $1(0)$ & 5312 \\
\hline 2018 & $64(1.4)$ & 2682 (58.6) & 493 (10.8) & 4021 (87.9) & $61(1.3)$ & 4575 \\
\hline
\end{tabular}


Table 4 Number of completed questionnaires, questionnaire precision (person separation and person reliability) and targeting

\begin{tabular}{lllll}
\hline Year & N (completed questionnaires) & Person separation & Person reliability & $\begin{array}{l}\text { Targeting: person } \\
\text { mean score (item =0) }\end{array}$ \\
\hline 2008 & 1690 & 2.57 & 0.87 & -1.92 \\
2009 & 6046 & 2.67 & 0.88 & -1.87 \\
2010 & 7630 & 2.68 & 0.88 & -1.81 \\
2011 & 7028 & 2.72 & 0.88 & -1.69 \\
2012 & 7432 & 2.61 & 0.87 & -1.87 \\
2013 & 7332 & 2.54 & 0.87 & -2.07 \\
2014 & 9202 & 2.58 & 0.87 & -2.08 \\
2015 & 8968 & 2.57 & 0.87 & -2.10 \\
2016 & 8944 & 2.54 & 0.87 & -2.19 \\
2017 & 10,624 & 2.55 & 0.87 & -2.16 \\
2018 & 9150 & 2.45 & 0.86 & -2.31
\end{tabular}

included posterior capsular break with or without vitreous loss. All independent variables were significantly related to the postoperative person Rasch score.

The same analysis was also conducted for each single year cohort separately. Postoperative visual acuity in the operated eye was significantly related to the postoperative person Rasch score in all single study years. Ocular co-morbidity and second-eye surgery were also significantly related to postoperative person Rasch score in all years except 2008. The other independent variables were significantly related to postoperative person Rasch score occasionally for single years.

\section{Discussion}

The relationship between demographic data, risk factors (co-existing eye diseases and surgical difficulty), first- or second-eye surgery, and Rasch scoring will reflect how the performance of the Catquest-9SF is influenced by changing patient cohorts. The annual cohorts showed changes in preoperative visual acuity and risk factors (Table 2). Mean age and proportion of women reported in the study both decreased over the years, in line with previous results [1]. A better preoperative visual acuity over time for patients undergoing cataract surgery has been documented in other cohorts [9]. Our study

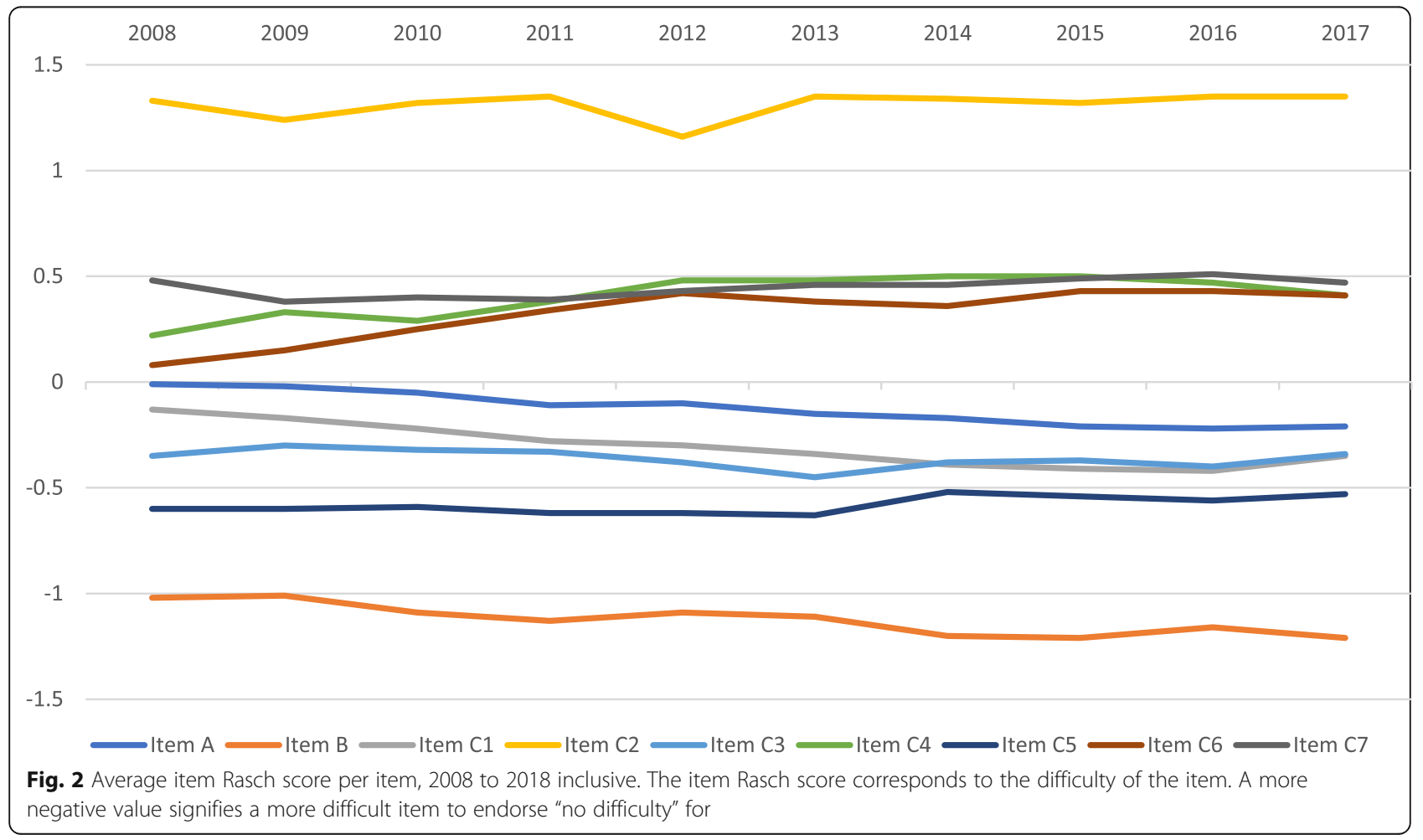




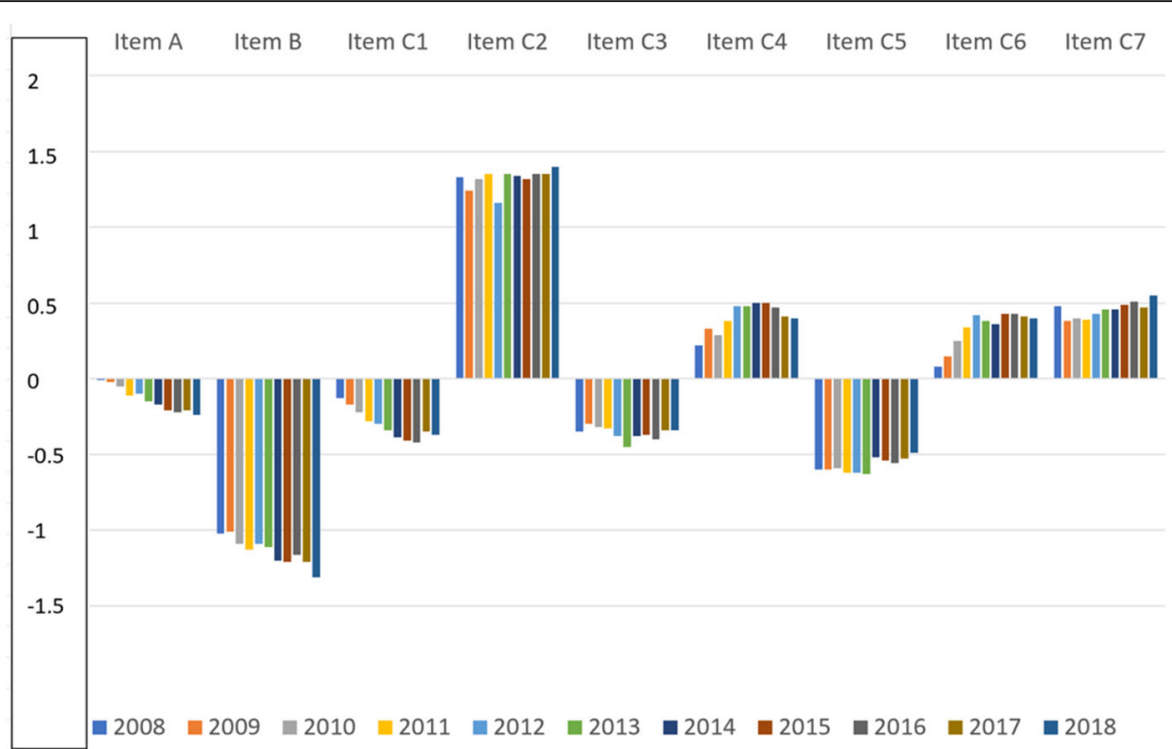

Fig. 3 Item average scoring per year, 2008 to 2018 inclusive. The figure shows how the level of difficulty for each item has performed during the study period

cohorts showed a decreasing frequency of second-eye surgery ( $p<0.001$, univariate analysis of variance) and an increasing frequency of ocular co-morbidity $(p<0.001$, univariate analysis of variance). The other risk factors in terms of surgical difficulties (data not shown) did not show any conclusive trend.

The precision of the Catquest-9SF remained acceptable over time (Table 4). The person separation coefficient should be over 2.0 for an acceptable precision [2, $4,10]$, and this limit was surpassed every year with a reasonable margin. Targeting, on the other hand, showed an increasing discrepancy between item mean difficulty (set to zero) and the mean person ability. The mean person ability changed from -1.92 to -2.31 over time, indicating an increasing ability for the patients to endorse the items. This is partly due to postoperative questionnaires being included in the analysis as a successful cataract extraction resolves all activity limitations caused by cataract, but probably also indicates an increasing visual acuity over time after surgery (Table 1). Figures 2 and 3 show the background for an increasingly poor targeting over time; item $\mathrm{A}$, item $\mathrm{B}$ and item

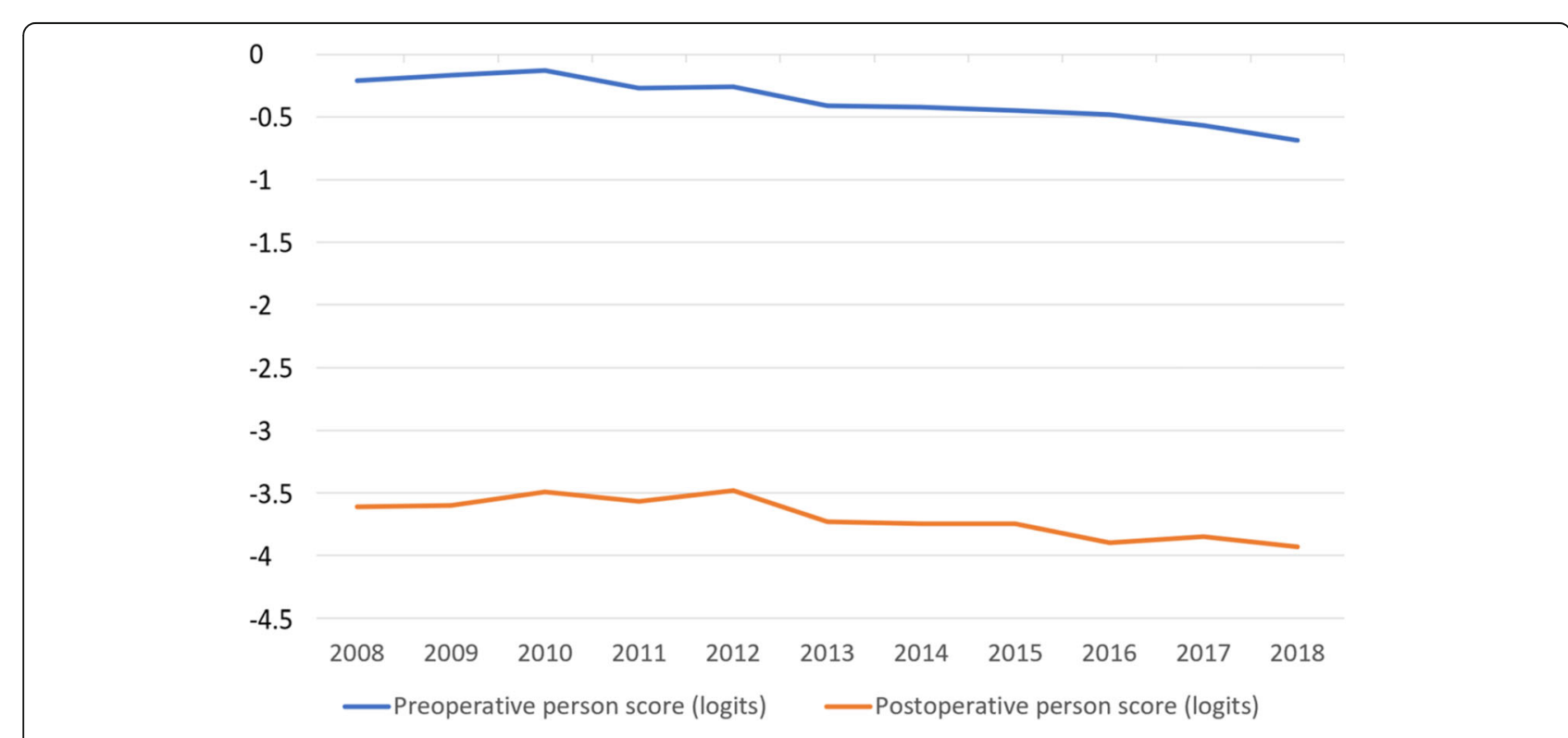

Fig. 4 Average person Rasch score before surgery (blue) and after surgery (yellow) over time. A more negative value means less activity limitation 
Table 5 Linear stepwise regression analysis of all data, 2008 to 2018 inclusive

\begin{tabular}{lllllll}
\hline Variable & Unstandardized beta & Standard Error & Standardized beta & Significance & $\mathbf{9 5 \%} \mathbf{C l}$, lower & $\mathbf{9 5 \%} \mathbf{C l}$, upper \\
\hline BCVA in the better eye & 3.399 & 0.053 & 0.320 & 0.000 & 3.294 & 3.503 \\
Age & -0.033 & 0.001 & -0.138 & 0.000 & -0.036 & -0.031 \\
Sex & -0.406 & 0.019 & -0.096 & 0.000 & -0.444 & -0.368 \\
Surgery year & -0.047 & 0.003 & -0.066 & 0.000 & -0.053 & -0.040 \\
Ocular comorbidity $^{a}$ & 0.211 & 0.020 & 0.049 & 0.000 & 0.172 & 0.251 \\
Surgical difficulty $^{\text {b }}$ & 0.216 & 0.037 & 0.027 & 0.000 & 0.143 & 0.289 \\
Second-eye surgery & -0.109 & 0.021 & -0.026 & 0.000 & -0.149 & -0.068 \\
\hline
\end{tabular}

Dependent variable: preoperative person Rasch score. Independent variables: preoperative BCVA (logMAR) in the better eye, age, sex (coded as female 0, male 1), surgery year, ocular co-morbidity (coded as yes 1 , no 0 ), surgical difficulty (coded as yes 1 , no 0 ) and second-eye surgery (coded as yes 1 , no 0 )

$B C V A=$ best corrected visual acuity; $\log M A R=$ logarithm of the minimum angle of resolution; $95 \% \mathrm{Cl}=95 \%$ confidence interval

${ }^{a}$ Ocular comorbidity includes age-related macular degeneration, glaucoma, diabetic retinopathy, and "other" sight-threatening ocular comorbidity

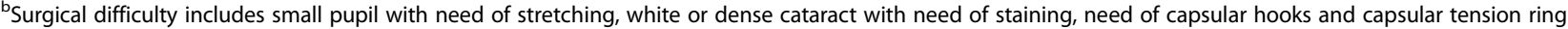

C1 show a statistically significant $(p<0.001)$ change in Rasch scoring, suggesting that these items are increasingly easier for the respondents to endorse. One way to counteract this development could be to include more difficult items in a future revision of the questionnaire. One area that has been suggested is the performance of several activities involving electronic devices which have become routine in recent years [11]. Many activity limitation cataract surgery questionnaires show low levels of difficulties pre-surgery, reflecting the fact that many of these questions were designed two to three decades ago when the surgery was performed at later stages of the disease as compared with present times. Rasch analysis provides insight into question targeting and has been used to re-validate most cataract surgery questionnaires [12]. Another way to counteract the poor targeting is to use item-banking [13, 14]. Other Rasch analysis outputs such as ordered response options and, dimensionality are not reported in the present article, but for each year they were within acceptable limits, as has been described in previous studies among different combinations of these cohorts [2, $15-18]$.
Some item scores converged to clusters over time (Fig. 2). The easiest item was item $\mathrm{C} 2$ (recognizing faces). Easy items were item C4 (walking on uneven ground), item C6 (reading subtitles on TV), and item C7 (doing hobby activities). More difficult items were item $\mathrm{C} 1$ (reading newspapers), item A (having problems in general), item C3 (seeing prices), and item C5 (doing handicrafts). The most difficult item was item B (satisfaction with vision). This means that items for near vision formed one cluster and items for distance vision formed another cluster over time.

The difficulty (item score) of the 9 items showed a similar pattern over the 11 study years (Fig. 3 ).

The average activity limitations for subjects (person Rasch score) in the annual cohorts gradually diminished over time (Fig. 4) both before surgery (blue line) and after surgery (yellow line). We believe that one reason for this change over time was better visual acuity (Tables 2 and 3 ).

To test the criterion validity of the Catquest-9SF, a comparison with other similar questionnaires would be desirable. However, no other questionnaires were used for these cohorts during the study period. One alternative is

Table 6 Linear stepwise regression analysis of all data, 2008-2018 inclusive

\begin{tabular}{|c|c|c|c|c|c|c|}
\hline Variable & Unstandardized beta & Standard error & Standardized beta & Significance & $95 \% \mathrm{Cl}$, lower & $95 \% \mathrm{Cl}$, upper \\
\hline BCVA in the operated eye & 4.138 & 0.063 & 0.331 & 0.000 & 4.014 & 4.262 \\
\hline Ocular comorbidity ${ }^{a}$ & 0.414 & 0.024 & 0.087 & 0.000 & 0.367 & 0.461 \\
\hline Second-eye surgery & -0.362 & 0.023 & -0.076 & 0.000 & -0.406 & -0.317 \\
\hline Sex & -0.178 & 0.023 & -0.038 & 0.000 & -0.222 & -0.134 \\
\hline Capsule complication & 0.483 & 0.122 & 0.019 & 0.000 & 0.244 & 0.722 \\
\hline Age & -0.004 & 0.001 & -0.013 & 0.007 & -0.006 & -0.001 \\
\hline Surgical difficulty ${ }^{b}$ & -0.112 & 0.044 & -0.012 & 0.011 & -0.198 & -0.026 \\
\hline
\end{tabular}

Dependent variable: postoperative person Rasch score. Independent variables: postoperative best corrected visual acuity (logMAR) in the operated eye, age, sex (coded as female 0 , male 1), ocular co-morbidity (coded as yes 1 , no 0 ), surgical difficulty (coded as yes 1 , no 0 ), second-eye surgery (coded as yes 1 , no 0 ), and capsule complication (coded as yes 1 , no 0 )

$B C V A=$ best corrected visual acuity; logMAR = logarithm of the minimum angle of resolution; $95 \% \mathrm{Cl}=95 \%$ confidence interval ${ }^{a}$ Ocular comorbidity includes age-related macular degeneration, glaucoma, diabetic retinopathy, and "other" sight threatening ocular comorbidity

${ }^{b}$ Surgical difficulty includes small pupil with need of stretching, white or dense cataract with need of staining, need of capsular hooks and capsular tension ring 
to make a comparison with clinical data; specifically, testing the correlation between Catquest-9SF scoring and visual acuity could be used as a proxy for similar questionnaires. Catquest-9SF measures activity limitations in daily life because of poor vision and so visual acuity is the most natural parameter for comparison with the person Rasch score for determining criterion validity. The Pearson correlation coefficient showed a significant correlation between preoperative visual acuity of the eye to be operated on and the preoperative person Rasch score. This correlation was even stronger between preoperative visual acuity of the better eye and preoperative Rasch score. This finding corresponds well with what was found in the first Catquest-9SF test in Sweden [19].

As shown in Table 5, the regression analysis indicated that preoperative visual acuity in the better eye was the most influential parameter on preoperative person Rasch score. However, other patient characteristics were also related to the preoperative person Rasch score in the total study cohort (Table 5) and for some single-year cohorts. Lower age, existence of an ocular co-morbidity, female sex, first-eye surgery, and existence of eye characteristics that may cause surgical difficulty were all related to higher Rasch person score (i.e., a less negative numeric value), indicating more perceived problems in daily life compared with the opposite situation.

The postoperative person Rasch score was significantly related to postoperative visual acuity of the operated eye (Table 6), and for single years it was also related to ocular co-morbidity and second-eye surgery. Age, sex, and surgical difficulties were less closely related to the postoperative person Rasch score. The relationship between ocular co-morbidity and poor patient-reported outcome of activity limitations has also been described for another questionnaire [20]. In recent years, cataract surgery has offered an opportunity to treat refractive errors by use of non-monofocal intraocular lenses. This makes another dimension interesting for patient-reported outcomes, namely visual symptoms [21].

A weakness of our study is the selection of clinics and patients based on one calendar month and on a voluntary participation. Another weakness is that we do not know what information are hidden among the nonresponders, which may affect the generalizability of our findings. A strength is the large number of completed questionnaires over a decade.

\section{Conclusion}

The Catquest-9SF had stable and reliable psychometric properties over 11 years of use, although the annual patient cohorts showed slightly improving mean visual acuity and slightly decreasing mean age. Future improvement could be achieved by introducing new items to replace groups of old items with similar properties. On the other hand, reducing the number of items too far may influence the precision, which is an important quality for a questionnaire [22].

\section{Acknowledgements}

Not applicable.

\section{Authors' contributions}

$M L$ performed the statistical analyses and was a major contributor in writing the manuscript. KP supervised the Rasch calculations. AB made the data available. All authors read and approved the final manuscript.

\section{Funding}

This study was financed by the Swedish Association of Local Authorities and Regions.

\section{Availability of data and materials}

The datasets generated and analysed during the current study are not publicly available due to integrity issues but are available from the corresponding author on reasonable request.

Ethics approval and consent to participate

This study was approved by the Swedish Ethical Board (registration number: 2019-03451).

Consent for publication

Not applicable.

\section{Competing interests}

The authors declare that they have no competing interests.

\section{Author details}

${ }^{1}$ Department of Clinical Sciences/Ophthalmology, Faculty of Medicine, Lund University, Trossögatan 4, 37137 Karlskrona, Lund, Sweden. Department of Clinical Neuroscience, Division of Ophthalmology and Vision, Karolinska Institutet, Stockholm, Sweden. ${ }^{3}$ St Erik Eye Hospital, Stockholm, Sweden. ${ }^{4}$ Capio Medocular, Lund, Sweden. ${ }^{5}$ Department of Clinical Neuroscience, Institute of Neuroscience and Physiology, Sahlgrenska Academy at the University of Gothenburg, Gothenburg, Sweden. ${ }^{6}$ University of New South Wales, Adelaide, SA, Australia. ${ }^{7}$ Department of Clinical Sciences/

Ophthalmology, Umeå University, Umeå, Sweden.

Received: 13 May 2020 Accepted: 1 November 2020

Published online: 01 December 2020

\section{References}

1. Behndig A, Montan P, Stenevi U, Kugelberg M, Lundström M. One million cataract surgeries: Swedish National Cataract Register 1992-2009. J Cataract Refract Surg. 2011;37(8):1539-45.

2. Lundström M, Pesudovs K. Catquest-9SF patient outcomes questionnaire: nine-item short-form Rasch-scaled revision of the Catquest questionnaire. J Cataract Refract Surg. 2009;35(3):504-13.

3. McAlinden C, Gothwal VK, Khadka J, Wright TA, Lamoureux EL, Pesudovs K. A head-to-head comparison of 16 cataract surgery outcome questionnaires. Ophthalmology. 2011;118(12):2374-81.

4. Khadka J, McAlinden C, Pesudovs K. Quality assessment of ophthalmic questionnaires: review and recommendations. Optom Vis Sci. 2013;90(8): 720-44.

5. Kabanovski A, Hatch W, Chaudhary V, El-Defrawy S, Reid R, Ahmed IIK, et al. Validation and application of Catquest-9SF in various populations: a systematic review. Surv Ophthalmol. 2020;65(3):348-60.

6. Mahmud I, Kelley T, Stowell C, Haripriya A, Boman A, Kossler I, et al. A proposed minimum set of outcome measures for cataract surgery. JAMA Ophthalmol. 2015;133(11):1247-52.

7. Lundström M, Barry P, Brocato L, Fitzpatrick C, Henry Y, Rosen P, et al. European registry for quality improvement in cataract surgery. Int J Health Care Qual Assur. 2014;27(2):140-51.

8. Lundström M, Goh P-P, Henry Y, Salowi MA, Barry P, Manning S, et al. The changing pattern of cataract surgery indications: a 5-year study of 2 cataract surgery databases. Ophthalmology. 2015;122(1):31-8. 
9. Behndig A, Lundström M, Serring I, Montan P, Kugelberg M, Nilsson I. Svensk Kataraktkirurgi. Årsrapport 2017 baserad på data från Nationella Kataraktregistret. (Swedish National Cataract Register Annual Report 2017). Nationella Kataraktregistret, Blekingesjukhuset, Karlskrona. In Swedish. www. kataraktreg.se.

10. Bond TG, Fox CM. Applying the Rasch model: fundamental measurement in the human sciences. 2nd ed. New York: Routledge, Taylor \& Francis Group; 2007.

11. Ribeiro F, Cochener B, Kohnen T, Mencucci R, Katz G, Lundstrom M, et al. Definition and clinical relevance of the concept of functional vision in cataract surgery ESCRS Position Statement on Intermediate Vision: ESCRS Functional Vision Working Group. J Cataract Refract Surg. 2020;46(Suppl 1): S1-3.

12. Gothwal VK, Wright TA, Lamoureux EL, Pesudovs K. The impact of cataract surgery questionnaire: re-evaluation using Rasch analysis. Acta Ophthalmol. 2011;89(5):423-8.

13. Pesudovs $\mathrm{K}$. Item banking: a generational change in patient-reported outcome measurement. Optom Vis Sci. 2010;87(4):285-93.

14. Khadka J, Fenwick E, Lamoureux E, Pesudovs K. Methods to develop the Eye-tem Bank to measure ophthalmic quality of life. Optom Vis Sci. 2016; 93(12):1485-94.

15. Rönbeck M, Lundström M, Kugelberg M. Study of possible predictors associated with self-assessed visual function after cataract surgery. Ophthalmology. 2011:118(9):1732-8

16. Grimfors $M$, Mollazadegan K, Lundström M, Kugelberg M. Ocular comorbidity and self-assessed visual function after cataract surgery. J Cataract Refract Surg. 2014;40(7):1163-9.

17. Mollazadegan K, Lundström M. A study of the correlation between patientreported outcomes and clinical outcomes after cataract surgery in ophthalmic clinics. Acta Ophthalmol. 2015;93(3):293-8.

18. Grimfors $M$, Lundström M, Höijer J, Kugelberg M. Intraoperative difficulties, complications and self-assessed visual function in cataract surgery. Acta Ophthalmol. 2018;96(6):592-9.

19. Lundström M, Behndig A, Kugelberg M, Montan P, Stenevi U, Pesudovs K. The outcome of cataract surgery measured with the Catquest-9SF. Acta Ophthalmol. 2011;89(8):718-23.

20. Schein OD, Steinberg EP, Cassard SD, Tielsch JM, Javitt JC, Sommer A. Predictors of outcome in patients who underwent cataract surgery. Ophthalmology. 1995;102(5):817-23.

21. Grzybowski A, Kanclerz P, Muzyka-Woźniak M. Methods for evaluating quality of life and vision in patients undergoing lens refractive surgery Graefes Arch Clin Exp Ophthalmol. 2019;257(6):1091-9.

22. Verhaar $\mathrm{HJ}$, Raymakers J. The precision and accuracy of questionnaires for clinical assessment. Age Ageing. 2001;30(4):275.

Ready to submit your research? Choose BMC and benefit from:

- fast, convenient online submission

- thorough peer review by experienced researchers in your field

- rapid publication on acceptance

- support for research data, including large and complex data types

- gold Open Access which fosters wider collaboration and increased citations

- maximum visibility for your research: over $100 \mathrm{M}$ website views per year

At BMC, research is always in progress.

Learn more biomedcentral.com/submissions 